\title{
Visualization of indicators to enhance the internal quality assurance system in Japanese universities
}

\author{
Susumu Shibui \\ National Institution for Academic Degrees and Quality Enhancement of Higher \\ Education
}

\begin{abstract}
To maintain the quality of higher education globally, it is important to standardize the indicators and evidence used in accreditation standards in each country. In this study, we investigated the indicators and evidence to evaluate the effectiveness of the Internal Quality Assurance (IQA) system. In Japan, as well as other countries, the main attention of accreditation has been directed to the IQA-based approach. However, little is known about the practical indicators and evidence to evaluate the IQA system. This study exploratory examined the indicators and evidence for measuring the IQA system using text-mining approach. We put focus on the past selfevaluation reports of the Certified Evaluation and Accreditation published by the National Institution for Academic Degrees and Quality Enhancement of Higher Education, one of Japan's accreditation institutions, between FY2012 and FY2018. Text that referred to the IQA system was extracted using morphological analysis. The data consists of text, including the rationales behind the opinions expressed by the 124 universities in Japan on the issues faced during the IQA system development process. Cluster analysis and self-organizing maps derived from the text-mining data revealed the similarity of frequently referred indicators and evidence for measuring the effectiveness of the IQA system. These results provide some insight into building the global accreditation standards as well as the basic indicators for measuring the effectiveness of the IQA system.
\end{abstract}

Keywords: accreditation, university evaluation, text-mining, quality assurance Themes: Higher Education, Assessment and Evaluation 
